\title{
SOME APPLICATIONS OF MINIMAL OPEN SETS
}

\author{
FUMIE NAKAOKA and NOBUYUKI ODA
}

(Received 16 January 2001)

\begin{abstract}
We characterize minimal open sets in topological spaces. We show that any nonempty subset of a minimal open set is pre-open. As an application of a theory of minimal open sets, we obtain a sufficient condition for a locally finite space to be a preHausdorff space.
\end{abstract}

2000 Mathematics Subject Classification. 54A05, 54D99.

1. Introduction. Let $X$ be a topological space. We call a nonempty open set $U$ of $X$ a minimal open set when the only open subsets of $U$ are $U$ and $\varnothing$.

In this paper, we study fundamental properties of minimal open sets and apply them to obtain some results on pre-open sets (cf. [2]) and pre-Hausdorff spaces.

In Section 2, we characterize minimal open sets, that is, we show that a nonempty open set $U$ is a minimal open set if and only if $\mathrm{Cl}(U)=\mathrm{Cl}(S)$ for any nonempty subset $S$ of $U$. This result implies that any nonempty subset $S$ of a minimal open set $U$ is a pre-open set.

In Section 3, we study minimal open sets in locally finite spaces. The results of this section are closely related to the work of James [1], and these results will be used in the next scetion.

In Section 4, we apply the theory of minimal open sets to study pre-open sets. Our first main result of this section is a property of the set of all minimal open sets in any nonempty finite open set which is not a minimal open set. This result enables us to prove a generalization of Theorem 2.5, when $U$ is a nonempty finite open set, in Theorem 4.4. Theorem 4.5 shows that our theory of minimal open set is useful to study pre-open sets.

Finally, we show that some conditions on minimal open sets implies pre-Hausdorffness of a space, that is, if any minimal open set of a locally finite space $X$ has two elements at least, then $X$ is a pre-Hausdorff space.

2. Minimal open sets. Let $(X, \tau)$ be a topological space.

Definition 2.1. A nonempty open set $U$ of $X$ is said to be a minimal open set if and only if any open set which is contained in $U$ is $\varnothing$ or $U$.

LEMMA 2.2. (1) Let $U$ be a minimal open set and $W$ an open set. Then $U \cap W=\varnothing$ or $U \subset W$.

(2) Let $U$ and $V$ be minimal open sets. Then $U \cap V=\varnothing$ or $U=V$. 
Proof. (1) Let $W$ be an open set such that $U \cap W \neq \varnothing$. Since $U$ is a minimal open set and $U \cap W \subset U$, we have $U \cap W=U$. Therefore $U \subset W$.

(2) If $U \cap V \neq \varnothing$, then we see that $U \subset V$ and $V \subset U$ by (1). Therefore $U=V$.

Proposition 2.3. Let $U$ be a minimal open set. If $x$ is an element of $U$, then $U \subset W$ for any open neighborhood $W$ of $x$.

Proof. Let $W$ be an open neighborhood of $x$ such that $U \not \subset W$. Then $U \cap W$ is an open set such that $U \cap W \subsetneq U$ and $U \cap W \neq \varnothing$. This contradicts our assumption that $U$ is a minimal open set.

Proposition 2.4. Let $U$ be a minimal open set. Then

$$
U=\cap\{W \mid W \text { is an open neighborhood of } x\}
$$

for any element $x$ of $U$.

Proof. By Proposition 2.3 and the fact that $U$ is an open neighborhood of $x$, we have $U \subset \cap\{W \mid W$ is an open neighborhood of $x\} \subset U$. Therefore we have the result.

THEOREM 2.5. Let $U$ be a nonempty open set. Then the following three conditions are equivalent:

(1) $U$ is a minimal open set.

(2) $U \subset \mathrm{Cl}(S)$ for any nonempty subset $S$ of $U$.

(3) $\mathrm{Cl}(U)=\mathrm{Cl}(S)$ for any nonempty subset $S$ of $U$.

Proof. $(1) \Rightarrow(2)$. Let $S$ be any nonempty subset of $U$. By Proposition 2.3, for any element $x$ of $U$ and any open neighborhood $W$ of $x$, we have

$$
S=U \cap S \subset W \cap S .
$$

Then, we have $W \cap S \neq \varnothing$ and hence $x$ is an element of $\mathrm{Cl}(S)$. It follows that $U \subset \mathrm{Cl}(S)$.

(2) $\Rightarrow(3)$. For any nonempty subset $S$ of $U$, we have $\mathrm{Cl}(S) \subset \mathrm{Cl}(U)$. On the other hand, by (2), we see $\mathrm{Cl}(U) \subset \mathrm{Cl}(\mathrm{Cl}(S))=\mathrm{Cl}(S)$. Therefore we have $\mathrm{Cl}(U)=\mathrm{Cl}(S)$ for any nonempty subset $S$ of $U$.

(3) $\Rightarrow(1)$. Suppose that $U$ is not a minimal open set. Then there exists a nonempty open set $V$ such that $V \subsetneq U$ and hence there exists an element $a \in U$ such that $a \notin V$. Then we have $\mathrm{Cl}(\{a\}) \subset V^{c}$, the complement of $V$. It follows that $\mathrm{Cl}(\{a\}) \neq \mathrm{Cl}(U)$.

A subset $M$ of a space $(X, \tau)$ is called a pre-open set if $M \subset \operatorname{IntCl}(M)$. The family of all pre-open sets in $(X, \tau)$ will be denoted by $\mathrm{PO}(X, \tau)$, (cf. [2]).

A space $(X, \tau)$ is called pre-Hausdorff if for each $x, y \in X, x \neq y$ there exist subsets $U, V \in \mathrm{PO}(X, \tau)$ such that $x \in U, y \in V$, and $U \cap V=\varnothing$.

THEOREM 2.6. Let $U$ be a minimal open set. Then any nonempty subset $S$ of $U$ is a pre-open set.

Proof. By Theorem 2.5(2), we have $\operatorname{Int} U \subset \operatorname{IntCl}(S)$. Since $U$ is an open set, we have $S \subset U=\operatorname{Int}(U) \subset \operatorname{IntCl}(S)$. 
THEOREM 2.7. Let $U$ be a minimal open set and $M$ a nonempty subset of $X$. If there exists an open neighborhood $W$ of $M$ such that $W \subset \mathrm{Cl}(M \cup U)$, then $M \cup S$ is a pre-open set for any nonempty subset $S$ of $U$.

Proof. By Theorem 2.5(3), we have $\mathrm{Cl}(M \cup S)=\mathrm{Cl}(M) \cup \mathrm{Cl}(S)=\mathrm{Cl}(M) \cup \mathrm{Cl}(U)=$ $\mathrm{Cl}(M \cup U)$. Since $W \subset \mathrm{Cl}(M \cup U)=\mathrm{Cl}(M \cup S)$ by assumption, we have $\operatorname{Int}(W) \subset$ $\operatorname{IntCl}(M \cup S)$. Since $W$ is an open neighborhood of $M$, namely $W$ is an open set such that $M \subset W$, we have $M \subset W=\operatorname{Int}(W) \subset \operatorname{Int} C l(M \cup S)$. Moreover we have $\operatorname{Int}(U) \subset \operatorname{Int} C l(M \cup$ $U)$, for $\operatorname{Int}(U)=U \subset \mathrm{Cl}(U) \subset \mathrm{Cl}(M) \cup \mathrm{Cl}(U)=\mathrm{Cl}(M \cup U)$. Since $U$ is an open set, we have $S \subset U=\operatorname{Int} U \subset \operatorname{IntCl}(M \cup U)=\operatorname{Int} \operatorname{Cl}(M \cup S)$. Therefore $M \cup S \subset \operatorname{IntCl}(M \cup S)$.

COROLLARY 2.8. Let $U$ be a minimal open set and $M$ a nonempty subset of $X$. If there exists an open neighborhood $W$ of $M$ such that $W \subset \mathrm{Cl}(U)$, then $M \cup S$ is a pre-open set for any nonempty subset $S$ of $U$.

Proof. By assumption, we have $W \subset \mathrm{Cl}(M) \cup \mathrm{Cl}(U)=\mathrm{Cl}(M \cup U)$. So by Theorem 2.7, we see that $M \cup S$ is a pre-open set.

The condition of Theorem 2.7, namely $W \subset \mathrm{Cl}(M \cup S)$, does not necessarily imply the condition of Corollary 2.8, namely $W \subset \mathrm{Cl}(S)$. We have the following example.

EXAMPLE 2.9. Let $X=\{a, b, c, d\}$ with topology $\theta=\{\varnothing,\{d\},\{a, b\},\{a, b, c\},\{a, b, d\}$, $X\}, U=\{a, b\}$ and $M=W=\{d\}$. Then $W=\{d\} \subset \operatorname{Cl}(\{a, b\} \cup\{d\})=\operatorname{Cl}(M \cup U)$ and $W=\{d\} \not \subset \mathrm{Cl}(\{a, b\})=\mathrm{Cl}(U)$.

THEOREM 2.10. Let $U$ be a minimal open set and $x$ an element of $X-U$. Then $W \cap U=\varnothing$ or $U \subset W$ for any open neighborhood $W$ of $x$.

Proof. Since $W$ is an open set, we have the result by Lemma 2.2.

COROLlary 2.11. Let $U$ be a minimal open set and $x$ an element of $X-U$. Define $U_{x} \equiv \cap\{W \mid W$ is an open neighborhood of $x\}$. Then $U_{x} \cap U=\varnothing$ or $U \subset U_{x}$.

Proof. If $U \subset W$ for any open neighborhood $W$ of $x$, then $U \subset \cap\{W \mid W$ is an open neighborhood of $x$ \}. Therefore $U \subset U_{x}$. Otherwise there exists an open neighborhood $W$ of $x$ such that $W \cap U=\varnothing$. Then we have $U \cap U_{x}=\varnothing$.

3. Finite open sets. In this section, we study some properties of minimal open sets in finite open sets and locally finite spaces.

THEOREM 3.1. Let $V$ be a nonempty finite open set. Then there exists at least one (finite) minimal open set $U$ such that $U \subset V$.

Proof. If $V$ is a minimal open set, we may set $U=V$. If $V$ is not a minimal open set, then there exists an (finite) open set $V_{1}$ such that $\varnothing \neq V_{1} \subsetneq V$. If $V_{1}$ is a minimal open set, we may set $U=V_{1}$. If $V_{1}$ is not a minimal open set, then there exists an (finite) open set $V_{2}$ such that $\varnothing \neq V_{2} \subsetneq V_{1} \subsetneq V$. Continuing this process, we have a sequence of open sets

$$
V \supsetneq V_{1} \supsetneq V_{2} \cdots \supsetneq V_{k} \supsetneq \cdots
$$


Since $V$ is a finite set, this process repeats only finitely. Then, finally we get a minimal open set $U=V_{n}$ for some positive integer $n$.

A topological space is said to be a locally finite space if each of its elements is contained in a finite open set.

COROLLARY 3.2. Let $X$ be a locally finite space and $V$ a nonempty open set. Then there exists at least one (finite) minimal open set $U$ such that $U \subset V$.

Proof. Since $V$ is a nonempty set, there exists an element $x$ of $V$. Since $X$ is a locally finite space, we have a finite open set $V_{x}$ such that $x \in V_{x}$. Since $V \cap V_{x}$ is a finite open set, we get a minimal open set $U$ such that $U \subset V \cap V_{x} \subset V$ by Theorem 3.1.

THEOREM 3.3. Let $V_{\lambda}$ be an open set for any $\lambda \in \Lambda$ and $W$ a nonempty finite open set. Then $W \cap\left(\cap_{\lambda \in \Lambda} V_{\lambda}\right)$ is a finite open set.

Proof. We see that there exists an integer $n$ such that $W \cap\left(\cap_{\lambda \in \Lambda} V_{\lambda}\right)=W \cap$ $\left(\cap_{i=1}^{n} V_{\lambda_{i}}\right)$ and hence we have the result.

THEOREM 3.4. Let $V_{\lambda}$ be an open set for any $\lambda \in \Lambda$ and $W_{\mu}$ a nonempty finite open set for any $\mu \in \mathcal{M}$. Let $S=\cup_{\mu \in \mathcal{M}} W_{\mu}$. Then $S \cap\left(\cap_{\lambda \in \Lambda} V_{\lambda}\right)$ is an open set.

Proof. Since $W_{\mu}$ is a finite open set, by Theorem 3.3, we have $W_{\mu} \cap\left(\cap_{\lambda \in \Lambda} V_{\lambda}\right)$ is a finite open set for any $\mu \in M$. Since

$$
S \cap\left(\cap_{\lambda \in \Lambda} V_{\lambda}\right)=\left(\cup_{\mu \in \mathcal{M}} W_{\mu}\right) \cap\left(\cap_{\lambda \in \Lambda} V_{\lambda}\right)=\cup_{\mu \in \mathcal{M}}\left(W_{\mu} \cap\left(\cap_{\lambda \in \Lambda} V_{\lambda}\right)\right),
$$

we have the result.

COROLLARY 3.5 (see [1]). Any locally finite space is an Alexandroff space.

4. Applications. Let $U$ be a nonempty finite open set. We see, by Lemma 2.2 and Corollary 3.2, that there exists a positive integer $k$ such that $\left\{U_{1}, U_{2}, \ldots, U_{k}\right\}$ is the set of all minimal open sets in $U$. Then it satisfies the following two conditions:

(a) $U_{i} \cap U_{j}=\varnothing$ for any $i, j$ with $1 \leq i, j \leq k$, and $i \neq j$.

(b) If $U^{\prime}$ is a minimal open set in $U$, then there exists $i$ with $1 \leq i \leq k$ such that $U^{\prime}=U_{i}$.

THEOREM 4.1. Let $U$ be a nonempty finite open set which is not a minimal open set. Let $\left\{U_{1}, U_{2}, \ldots, U_{n}\right\}$ be the set of all minimal open sets in $U$ and $x$ an element of $U-\left(U_{1} \cup U_{2} \cup \cdots \cup U_{n}\right)$. Define $U_{x} \equiv \cap\{W \mid W$ is an open neighborhood of $x\}$. Then there exists a positive integer $i$ of $\{1, \ldots, n\}$ such that $U_{i} \subset U_{x}$.

Proof. Assume that $U_{i} \not \subset U_{x}$ for any positive integer $i$ of $\{1, \ldots, n\}$. Then we have $U_{i} \cap U_{x}=\varnothing$ for any minimal open set $U_{i}$ in $U$ by Corollary 2.11. Since $U_{x}$ is a nonempty finite open set by Theorem 3.3, there exists a minimal open set $U^{\prime}$ such that $U^{\prime} \subset U_{x}$ by Theorem 3.1. Since $U^{\prime} \subset U_{x} \subset U$, we have $U^{\prime}$ is a minimal open set in $U$. By assumption, we have $U_{i} \cap U^{\prime} \subset U_{i} \cap U_{x}=\varnothing$ for any minimal open set $U_{i}$. Therefore $U^{\prime} \neq U_{i}$ for any positive integer $i$ of $\{1,2, \ldots, n\}$. This contradicts our assumption. 
Proposition 4.2. Let $U$ be a nonempty finite open set which is not a minimal open set. Let $\left\{U_{1}, U_{2}, \ldots, U_{n}\right\}$ be the set of all minimal open sets in $U$ and $x$ an element of $U-\left(U_{1} \cup U_{2} \cup \cdots \cup U_{n}\right)$. Then there exists a positive integer $i$ of $\{1, \ldots, n\}$ such that $U_{i} \subset W_{x}$ for any open neighborhood $W_{x}$ of $x$.

Proof. Since $W_{x} \supset \cap\{W \mid W$ is an open neighborhood of $x\}$, we have the result by Theorem 4.1.

THEOREM 4.3. Let $U$ be a nonempty finite open set which is not a minimal open set. Let $\left\{U_{1}, U_{2}, \ldots, U_{n}\right\}$ be the set of all minimal open sets in $U$ and $x$ an element of $U-\left(U_{1} \cup U_{2} \cup \cdots \cup U_{n}\right)$. Then there exists a positive integer $i$ of $\{1, \ldots, n\}$ such that $x$ is an element of $\mathrm{Cl}\left(U_{i}\right)$.

Proof. By Proposition 4.2, there exists a positive integer $i$ of $\{1, \ldots, n\}$ such that $U_{i} \subset W$ for any open neighborhood $W$ of $x$. Therefore $U_{i} \cap W \supset U_{i} \cap U_{i} \neq \varnothing$ for any open neighborhood $W$ of $x$. Therefore we have the result.

The following result is a generalization of Theorem 2.5, when $U$ is a nonempty finite open set.

THEOREM 4.4. Let $U$ be a nonempty finite open set and $U_{i}$ a minimal open set in $U$ for each $i \in\{1,2, \ldots, n\}$. Then the following three conditions are equivalent:

(1) $\left\{U_{1}, U_{2}, \ldots, U_{n}\right\}$ is the set of all minimal open sets in $U$.

(2) $U \subset \mathrm{Cl}\left(S_{1} \cup S_{2} \cup \cdots \cup S_{n}\right)$ for any nonempty subsets $S_{i}$ of $U_{i}$ for $i \in\{1,2, \ldots, n\}$.

(3) $\mathrm{Cl}(U)=\mathrm{Cl}\left(S_{1} \cup S_{2} \cup \cdots \cup S_{n}\right)$ for any nonempty subsets $S_{i}$ of $U_{i}$ for $i \in\{1,2, \ldots, n\}$.

Proof. (1) $\Rightarrow(2)$. If $U$ is a minimal open set, then this is the result of Theorem 2.5(2). Otherwise $U$ is not a minimal open set. If $x$ is any element of $U-\left(U_{1} \cup U_{2} \cup \cdots \cup U_{n}\right)$, we have $x \in \operatorname{Cl}\left(U_{1}\right) \cup \mathrm{Cl}\left(U_{2}\right) \cup \cdots \cup \mathrm{Cl}\left(U_{n}\right)$ by Theorem 4.3. Therefore

$$
\begin{aligned}
U \subset \mathrm{Cl}\left(U_{1}\right) \cup \mathrm{Cl}\left(U_{2}\right) \cup \cdots \cup \mathrm{Cl}\left(U_{n}\right) & =\mathrm{Cl}\left(S_{1}\right) \cup \mathrm{Cl}\left(S_{2}\right) \cup \cdots \cup \mathrm{Cl}\left(S_{n}\right) \\
& =\mathrm{Cl}\left(S_{1} \cup S_{2} \cup \cdots \cup S_{n}\right)
\end{aligned}
$$

by Theorem 2.5(3).

(2) $\Rightarrow$ (3). For any nonempty subset $S_{i}$ of $U_{i}$ with $i \in\{1,2, \ldots, n\}$, we have $\operatorname{Cl}\left(S_{1} \cup S_{2} \cup\right.$ $\left.\cdots \cup S_{n}\right) \subset \mathrm{Cl}(U)$. On the other hand, by (2), we see

$$
\mathrm{Cl}(U) \subset \mathrm{Cl}\left(\mathrm{Cl}\left(S_{1} \cup S_{2} \cup \cdots \cup S_{n}\right)\right)=\mathrm{Cl}\left(S_{1} \cup S_{2} \cup \cdots \cup S_{n}\right) .
$$

Therefore we have $\mathrm{Cl}(U)=\mathrm{Cl}\left(S_{1} \cup S_{2} \cup \cdots \cup S_{n}\right)$ for any nonempty subset $S_{i}$ of $U_{i}$ with $i \in\{1,2, \ldots, n\}$.

(3) $\Rightarrow(1)$. Suppose that $V$ is a minimal open set in $U$ and $V \neq U_{i}$ for $i \in\{1,2, \ldots, n\}$. Then we have $V \cap \mathrm{Cl}\left(U_{i}\right)=\varnothing$ for each $i \in\{1,2, \ldots, n\}$. It follows that any element of $V$ is not contained in $\mathrm{Cl}\left(U_{1} \cup U_{2} \cup \cdots \cup U_{n}\right)$. This contradicts the condition (3) because $V \subset U \subset \mathrm{Cl}(U)=\mathrm{Cl}\left(S_{1} \cup S_{2} \cup \cdots \cup S_{n}\right)$.

Let $U$ be a nonempty finite open set, $\left\{U_{1}, U_{2}, \ldots, U_{n}\right\}$ the set of all minimal open sets in $U$ and $x_{i}$ an element of $U_{i}$ for each $i \in\{1,2, \ldots, n\}$. Then we see that the set $\left\{x_{1}, x_{2}\right.$, $\left.\ldots, x_{n}\right\}$ is a pre-open set by Theorem 4.4. Moreover, we have the following result. 
THEOREM 4.5. Let $U$ be a nonempty finite open set and $\left\{U_{1}, U_{2}, \ldots, U_{n}\right\}$ the set of all minimal open sets in $U$. Let $S$ be any subset of $U-\left(U_{1} \cup U_{2} \cup \cdots \cup U_{n}\right)$ and $S_{i}$ be any nonempty subset of $U_{i}$ for each $i \in\{1,2, \ldots, n\}$. Then $S \cup S_{1} \cup S_{2} \cdots \cup S_{n}$ is a pre-open set.

Proof. By Theorem 4.4(2), we have

$$
U \subset \mathrm{Cl}\left(S_{1} \cup S_{2} \cdots \cup S_{n}\right) \subset \mathrm{Cl}\left(S \cup S_{1} \cup S_{2} \cdots \cup S_{n}\right) .
$$

Since $U$ is an open set, then we have

$$
S \cup S_{1} \cup S_{2} \cdots \cup S_{n} \subset U=\operatorname{Int}(U) \subset \operatorname{IntCl}\left(S \cup S_{1} \cup S_{2} \cdots \cup S_{n}\right) .
$$

Then we have the result.

THEOREM 4.6. Let $X$ be a locally finite space. If any minimal open set of $X$ has two elements at least, then $X$ is a pre-Hausdorff space.

Proof. Let $x, y$ be elements of $X$ such that $x \neq y$. Since $X$ is a locally finite space, there exists finite open sets $U$ and $V$ such that $x \in U$ and $y \in V$. By Theorem 3.1, there exists the set $\left\{U_{1}, U_{2}, \ldots, U_{n}\right\}$ of all minimal open sets in $U$ and the set $\left\{V_{1}, V_{2}, \ldots, V_{m}\right\}$ of all minimal open sets in $V$.

CASE 1. If there exists $i$ of $\{1,2, \ldots, n\}$ and $j$ of $\{1,2, \ldots, m\}$ such that $x \in U_{i}$ and $y \in V_{j}$, then, by Theorem 2.6, $\{x\}$ and $\{y\}$ are disjoint pre-open sets which contains $x$ and $y$, respectively.

CASE 2. If there exists $i$ of $\{1,2, \ldots, n\}$ such that $x \in U_{i}$ and $y \notin V_{j}$ for any $j$ of $\{1,2, \ldots, m\}$, then we find an element $y_{j}$ of $V_{j}$ for each $j$ such that $\{x\}$ and $\left\{y, y_{1}\right.$, $\left.y_{2}, \ldots, y_{m}\right\}$ are pre-open sets and $\{x\} \cap\left\{y, y_{1}, y_{2}, \ldots, y_{n}\right\}=\varnothing$ by Theorems 2.6, 4.5 and the assumption.

CASE 3. If $x \notin U_{i}$ for any $i$ of $\{1,2, \ldots, n\}$ and $y \notin V_{j}$ for any $j$ of $\{1,2, \ldots, m\}$, then we find elements $x_{i}$ of $U_{i}$ and $y_{j}$ of $V_{j}$ for each $i, j$ such that $\left\{x, x_{1}, x_{2}, \ldots, x_{n}\right\}$ and $\left\{y, y_{1}, y_{2}, \ldots, y_{m}\right\}$ are pre-open sets and $\left\{x, x_{1}, x_{2}, \ldots, x_{n}\right\} \cap\left\{y, y_{1}, y_{2}, \ldots, y_{m}\right\}=\varnothing$ by Theorem 4.5 and the assumption. We remark that we use the assumption that any minimal open set of $X$ has at least two elements for the case $U_{i}=V_{j}$ for some $i$ and $j$ in the argument of cases (2) and (3).

Therefore $X$ is a pre-Hausdorff space.

\section{REFERENCES}

[1] I. M. James, Alexandroff spaces, Rend. Circ. Mat. Palermo (2) Suppl. (1992), no. 29, 475481, International Meeting on Topology in Italy (Italian) (Lecce, 1990/Otranto, 1990). MR 94g:54020. Zbl 793.54006.

[2] A. S. Mashhour, M. E. Abd El-Monsef, and S. N. El-Deep, On precontinuous and weak precontinuous mappings, Proc. Math. Phys. Soc. Egypt (1982), no. 53, 47-53. MR 87c:54002. Zbl 571.54011.

FUMIE NAKAOKA: DEPARTMENT OF APPLIED MATHEMATICS, FUKUOKA UNIVERSITY, NANAKUMA JONAN-KU, FUKUOKA 814-0180, JAPAN

E-mail address: fumi e@fukuoka-u.ac.jp

NOBUyUKi OdA: DEPARTMENT OF APPLIED MATHEMATICS, FUKUOKA UNIVERSITY, NANAKUMA JONAN-KU, FUKUOKA 814-0180, JAPAN

E-mail address: odanobu@cis.fukuoka-u.ac.jp 


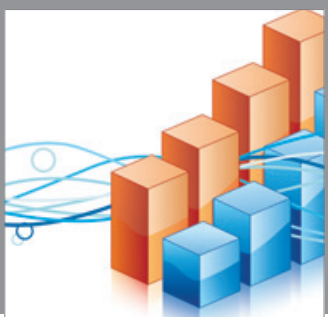

Advances in

Operations Research

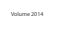

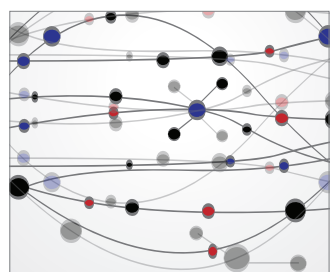

\section{The Scientific} World Journal
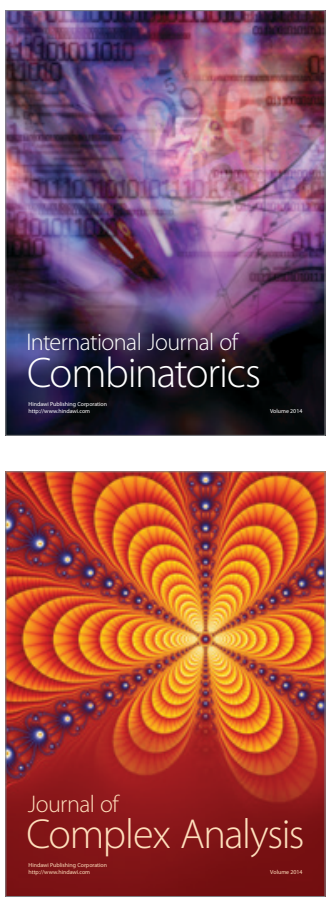

International Journal of

Mathematics and

Mathematical

Sciences
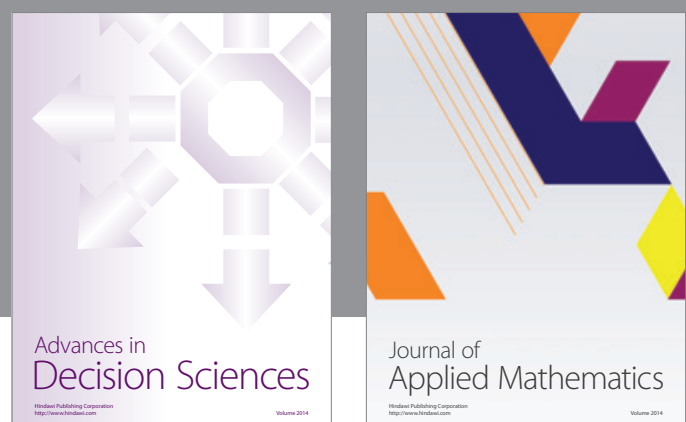

Journal of

Applied Mathematics
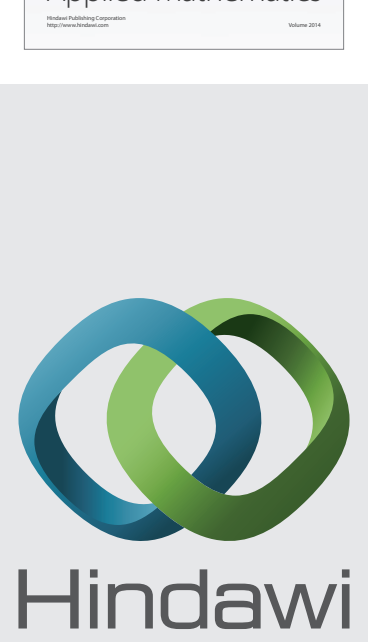

Submit your manuscripts at http://www.hindawi.com
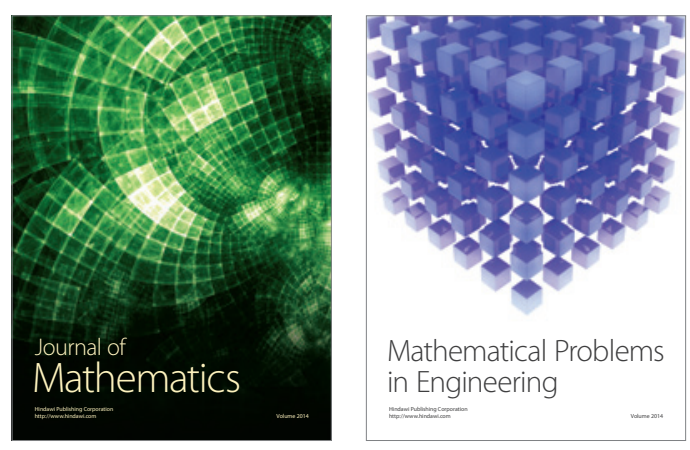

Mathematical Problems in Engineering
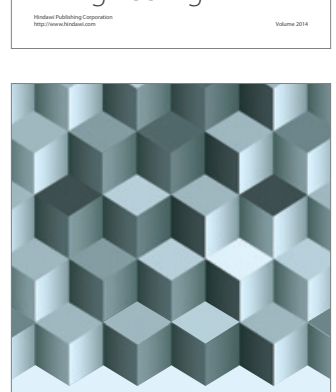

Journal of

Function Spaces
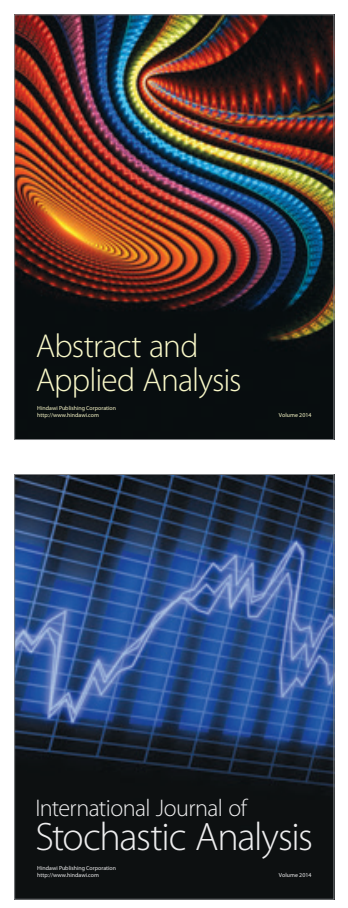

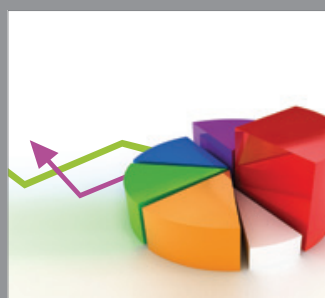

ournal of

Probability and Statistics

Promensencen
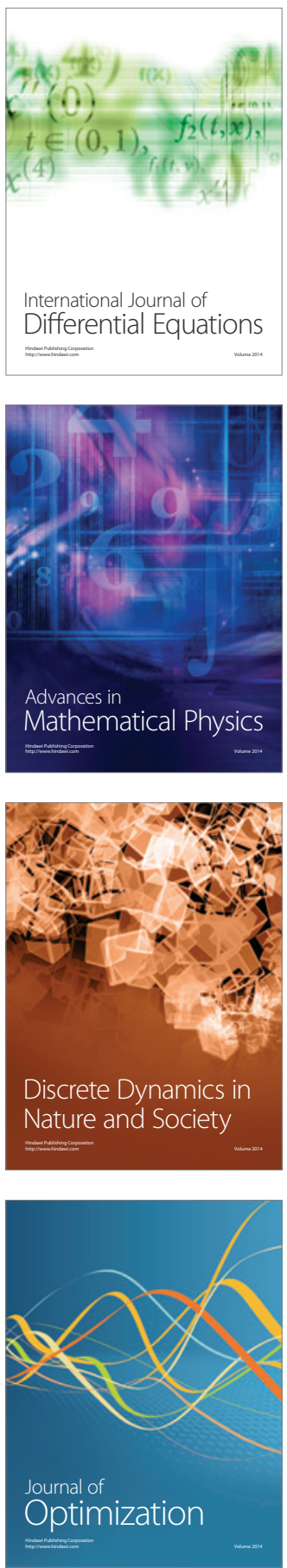